\title{
REVIEW
}

\section{Cognitive changes associated with ADT: a review of the literature}

\author{
Rhoda J Jamadar ${ }^{1}$, Mary J Winters ${ }^{1}$ and Pauline M Maki ${ }^{1,2}$
}

The use of androgen deprivation therapy (ADT) has increased since the early 1990s after early detection efforts and greater use of the prostate-specific antigen (PSA) test. Although ADT is associated with favorable clinical outcomes, ADT has been associated with an increased risk for cardiovascular disease, increased serum cholesterol, triglycerides, insulin resistance, body mass index and fat body mass. Here we review findings from 11 clinical studies examining the effects of ADT on cognition as measured by standardized tests in cognitive domains such as verbal and spatial memory. Most of these studies have important limitations, including small sample sizes, suboptimal control groups and baseline group differences in confounding factors. Despite these limitations, the best designed studies, those comparing patients on ADT to healthy controls, generally suggest that spatial memory might be especially sensitive to the effects of ADT. Critically, to date there is only one study involving random assignment of ADT versus close clinical observation. That study revealed a decrease in verbal memory with ADT, but was limited in sample size and did not include a measure of spatial memory. A recent observational study revealed no substantial evidence of cognitive impairment with ADT, even in the domain of verbal memory. Like the randomized study, however, this large observational study lacked a measure of spatial memory. Overall, the studies with the best controls suggest a potential negative impact of ADT on spatial memory, and perhaps verbal memory, and a need for continued investigation of the impact of ADT on cognition, particularly in these two cognitive domains.

Asian Journal of Andrology (2012) 14, 232-238; doi:10.1038/aja.2011.107; published online 20 February 2012

Keywords: androgen deprivation therapy; cognition; prostate cancer

\section{INTRODUCTION}

According to the World Health Organization (WHO) International Agency for Research on Cancer, prostate cancer is the second most frequently diagnosed cancer of men worldwide, with 899000 new cases in 2008 (13.6\% of the total), and is the fifth most common cancer overall. ${ }^{1}$ Documented incidence rates of prostate cancer differ considerably across countries worldwide because of the greater use of prostate-specific antigen (PSA) testing and subsequent biopsy in developed countries. The PSA test has a greater effect on incidence than mortality, so there is a 25 -fold difference in documented incidence rates worldwide, but only a 10 -fold difference in mortality rates. Asia has a low age-standardized mortality rate (2.5/100 000) compared with Australia (15.4/100 000) and the United States (9.7/100 000). Across Asia, rates are uniformly low, including in Japan (5/100 000), India (2.5/100 000) and China (1.8/100 000).

With greater use of the PSA test since the early 1990s, the use of androgen deprivation therapy (ADT) increased in the United States from $3.7 \%$ of patients to $31 \% .^{2}$ ADT includes pharmacological therapies and surgical bilateral orchiectomy. Gonadotropin-releasing hormone agonist ( $\mathrm{GnRHa}$ ) therapies account for the majority of ADT therapy. ${ }^{2,3}$ Pharmacological therapies include luteinizing hormonereleasing hormone (LHRH) and GnRHa therapies such as leuprolide (Lupron), goserelin (Zoladex) and triptorelin. Flutamide (oral antiandrogen) is often prescribed with GnRH analogs to block testosterone receptors from the initial surge of testosterone. ${ }^{4}$ Currently, in the United States, half of men with prostate cancer will receive ADT at some point after diagnosis. ${ }^{5,6} \mathrm{ADT}$ is primarily used among high-risk men and among men aged 65 and older. ${ }^{7}$ Given the large number of men on ADT, side effects are important to consider, especially since adverse effects and complications of treatment may impact health and quality of life more than the cancer itself. ${ }^{2,3}$

ADT has been associated with comorbidities such as an increased risk for cardiovascular disease, increased serum cholesterol, triglycerides, insulin resistance, body mass index and fat body mass. ${ }^{8}$

Recent reviews have highlighted these comorbidities, but have made minimal reference, if any, to cognitive consequences of ADT. ${ }^{8,9}$ Consideration of the cognitive effects of ADT is important since more than half of new prostate cancer diagnoses occur after age 65 years, an age associated with increased rates of cognitive aging. ${ }^{10}$ Understanding factors that might exacerbate the typical declines in cognitive function that occur in older men is important for maintaining a high level of social and occupational function and for maintaining quality of life. For example, neuropsychological measures of spatial ability and processing speed have been shown to predict driving errors both onroad and in driving simulators. ${ }^{11} \mathrm{~A}$ thorough review of the clinical literature was published in 2008 and indicated that although studies suffered from small sample sizes, there was a strong argument that ADT is linked to subtle but significant cognitive declines in men with

${ }^{1}$ Center for Cognitive Medicine, Department of Psychiatry, University of Illinois at Chicago, Chicago, IL 60612, USA and ${ }^{2}$ Department of Psychology, University of Illinois at Chicago, Chicago, IL 60612, USA 
prostate cancer. ${ }^{12}$ Since that publication, new high-quality evidence has emerged, ${ }^{13}$ warranting a reexamination of the data.

\section{EVIDENCE FOR COGNITIVE EFFECTS OF TESTOSTERONE}

Basic science studies have elucidated the structural and physiological consequences of androgen blockade in the brain. Androgen receptors are found in brain areas critical for memory and higher order cognitive functions, including the hippocampus, parietal lobe and prefrontal cortex. ${ }^{14}$ Especially relevant for studies of memory is the finding that androgen withdrawal leads to decreases in synaptic spine density in the CA1 region of the hippocampus, the brain region that subserves memory function, allowing the encoding of new memories. ${ }^{15,16}$ Androgen depletion via gonadectomy in rodents has been shown to increase brain levels of beta-amyloid protein, the neuropathological hallmark of Alzheimer's disease. Supplementation with $5 \alpha$-dihydrotestosterone, the active metabolite of testosterone, reverses this effect. ${ }^{17}$ In humans, the apolipoprotein epsilon 4 allele is associated with a greater risk for Alzheimer's disease. ${ }^{18}$ Androgen blockade in rodents expressing this allele has been shown to impair memory performance on the water maze task. ${ }^{19}$ Testosterone also exerts effects from the aromatization to estrogen which in turn binds to estrogen binding sites located in the hippocampus and prefrontal cortex. ${ }^{20}$ Estrogen withdrawal is associated with reductions in memory in both basic and clinical studies (see reviews by Maki and colleagues ${ }^{21,22}$ ).

Longitudinal studies indicate that men experience a gradual loss of testosterone as they age, but there are appreciable individual differences in the rate of loss. ${ }^{23-25}$ Neuropathological evidence suggests that testosterone levels in the brain also decrease with age. ${ }^{26}$ Prospective studies of male volunteers in the Baltimore Longitudinal Study of Aging demonstrate that men showing greater rates of decline in testosterone over a 10- to 20-year period also show the greatest rates of cognitive declines, the lowest rates of glucose metabolism in brain regions subserving cognition and the greatest risk of Alzheimer's disease. ${ }^{27-29}$ Serum levels of testosterone are reported to be lower in men with Alzheimer's disease compared to the controls, ${ }^{30}$ and men with a neuropathological diagnosis for Alzheimer's disease have lower brain levels of testosterone. ${ }^{11}$ Findings from randomized, placebocontrolled clinical trials of testosterone supplementation in older or hypogonadal men show variable cognitive effects. ${ }^{31-39}$

\section{METHODS}

A review was conducted through PubMed to identify studies investigating the cognitive effects of ADT. The search words 'androgen deprivation therapy, cognition, memory, imaging' were used to search for articles. References from articles found were cross-referenced to identify other relevant articles. The search revealed a total of 11 studies examining ADT in relation to objective cognitive performance. ${ }^{40-42}$ Our primary focus was effects on specific cognitive domains including verbal memory (e.g., memory for word lists), visual memory (e.g., memory for geometric designs), spatial memory (e.g., route memory), spatial abilities (e.g., mental rotations), attention, verbal fluency, processing speed, executive function (e.g., planning and mental flexibility) and working memory. Additionally, when available, we report findings based on a Reliable Change Index (RCI) calculation or a similar approach. Whereas analyses of typical scores compare group differences in mean scores, analyses of RCIs examine group differences in the percentage of patients and controls that show clinically significant impairment. We required that studies include standardized neuropsychological tests with appropriate normative data and precedence in the clinical literature. For that reason, we did not include studies that relied exclusively on experimental measures of cognitive performance ${ }^{43}$ which are not validated clinically and have no norms. Similarly, we did not review studies that included only global measures of cognition such as the total score on the High Sensitivity Cognitive Screen, ${ }^{44,45}$ because such measures were developed and validated to detect gross cognitive impairment as contrasted with more subtle cognitive difficulties within specific cognitive domains.

Below we first review nine studies that examine the effects of ADT on cognitive performance (Table 1). The simplest study design involves only one patient group whose cognitive performance is first assessed before ADT and again after receiving ADT for several weeks. These studies can provide insights into what cognitive changes patients might experience over the course of treatment but are limited because they provide no estimate from a control group about how performance should change over time. We review three studies with that simple design. ${ }^{40-42} \mathrm{~A}$ better study design is one where the patient groups are tested at baseline and after treatment and compared to a control group tested at two sessions spaced at equal intervals. Such a design allows an estimation of change in performance related to ADT given what would be expected in the absence of ADT. Ideally, the control group would be a patient group not receiving ADT, but most studies instead include a healthy comparison group, largely due to ethical concerns about withholding ADT. In that case, the study design does not control for the presence of prostate cancer and related issues that may impact cognitive function (e.g., psychological ramifications of diagnosis, fatigue, cancer treatments). We review three studies involving a healthy community control group ${ }^{46-48}$ and two studies that an untreated patient control group. ${ }^{13,49}$ Only one of these studies directly compared different ADT treatments, and treatment assignment was randomized in that study. ${ }^{50}$ In a follow-up publication, that randomized study added a healthy comparison group. ${ }^{49}$

In the second part of this review, we describe two studies examining the effects of estradiol treatment on cognition in men receiving ADT. The rationale for including these studies is that they provide insights into the potential therapeutic benefit of estrogen treatment and the extent to which ADT effects on estradiol might explain ADT-related changes in cognition.

\section{DOES ADT AFFECT COGNITIVE FUNCTION?}

\section{Studies examining patient groups only over time}

Almeida and colleagues ${ }^{41}$ investigated the effects of ADT in men with prostate cancer (mean age: 72 years) over one year. Patients were treated with flutamide $(250 \mathrm{mg})$ for 1 week and then began treatment with leuprolide for 36 weeks. They then discontinued ADT and were followed for an additional 18 weeks. Patients completed eight visits over a 1-year period, including tests 1 week before treatment (prebaseline), on the day they started flutamide (baseline), during treatment (i.e., 4, 12, 24 and 36 weeks after baseline) and after treatment discontinuation (i.e., 42, 48 and 54 weeks after baseline). Cognitive tests included measures of verbal memory, visual memory and spatial abilities, as well as a measure of global cognitive function. Results showed significantly improved performance on tests of verbal and visual memory over the 1-year period, both during and after ADT treatment. Such an improvement is expected over time given practice effects. Overall, there was little evidence of a negative impact of ADT on cognition. One exception was that CAMCOG scores improved significantly after treatment withdrawal, suggesting that ADT may have had some negative effects.

Salminen et al. ${ }^{40}$ conducted a prospective observational study of 23 men (mean age: 65 years) with non-metastatic prostate cancer receiving 
Table 1 Summary of study designs and cognitive outcomes

\begin{tabular}{|c|c|c|c|c|c|c|c|c|c|c|}
\hline Author & & $\begin{array}{l}\text { Almeida } \\
\text { et al. }^{41} \\
2004\end{array}$ & $\begin{array}{l}\text { Salminen } \\
\text { et al. }{ }^{40} \\
2005\end{array}$ & $\begin{array}{c}\text { Mohile and } \\
\text { Lacy }^{42} \\
2010\end{array}$ & $\begin{array}{c}\text { Cherrier } \\
\text { et al. }^{47} \\
2003\end{array}$ & $\begin{array}{l}\text { Jenkins } \\
\text { et al. }{ }^{46} \\
2005\end{array}$ & $\begin{array}{l}\text { Cherrier } \\
\text { et al. }^{48} \\
2009\end{array}$ & $\begin{array}{l}\text { Green } \\
\text { et al. }^{50} \\
2002\end{array}$ & $\begin{array}{l}\text { Green } \\
\text { et al. }^{49} \\
2004\end{array}$ & $\begin{array}{l}\text { Alibhai } \\
\text { et al. }^{13} \\
2010\end{array}$ \\
\hline \multirow[t]{3}{*}{ Testing Timeline } & Pre treatment baseline & $x$ & $x$ & $x$ & $x$ & $x$ & $x$ & $x$ & $x$ & $x$ \\
\hline & ADT & $x$ & $x$ & $x$ & $x$ & $x$ & $x$ & $x$ & - & $x$ \\
\hline & Washout & $x$ & - & - & $x$ & $x$ & - & - & - & - \\
\hline \multirow{4}{*}{$\begin{array}{l}\text { Design: } \\
N \text { (initial enrollment) }\end{array}$} & Randomized & - & - & - & - & - & - & x & $x$ & - \\
\hline & ADT group & $37(44)$ & 23 & 21(32) & 19 & $32(36)$ & $19(21)$ & $50\left(82^{a}\right)$ & $48\left(82^{a}\right)$ & $69(77)$ \\
\hline & CG - patients, no treatment & - & - & - & - & - & - & 15 & 42 & $75(82)$ \\
\hline & CG - healthy & - & - & - & 15 & 18 & $21(24)$ & - & $15(20)$ & $74(82)$ \\
\hline \multirow[t]{5}{*}{ ADT groups } & Leuprolide & $x$ & $x$ & Not reported & $x$ & - & X & $x$ & X & Not reported \\
\hline & Cyproterone acetate & - & - & Not reported & - & $x$ & - & $x$ & $x$ & Not reported \\
\hline & Goserelin & - & - & Not reported & - & $x$ & - & $x$ & $x$ & Not reported \\
\hline & Age (whole sample) & 72 & 65 & 71 & 65 & 67 & 64 & 73 & 73 & 69 \\
\hline & Education (year) & 14.9 & 8.5 & 15 & 16 & - & 16.9 & 9.1 & $10.1^{*}$ & 16 \\
\hline \multirow[t]{13}{*}{ Cognitive Measures } & See text for $\mathrm{RCl}$ & - & - & $x$ & $x$ & $x$ & - & $x$ & - & $x$ \\
\hline & Verbal memory & + & + & 0 & $\boldsymbol{\varphi}^{\mathrm{b}}$ & 0 & 0 & $\uparrow \downarrow$ & $\downarrow$ & 0 \\
\hline & Visual memory & + & 0 & 0 & - & 0 & - & $\downarrow$ & $\downarrow$ & 0 \\
\hline & Spatial memory & - & 0 & - & $\downarrow$ & 0 & $\downarrow$ & - & - & - \\
\hline & Spatial ability & 0 & 0 & - & 0 & 0 & $\downarrow$ & - & - & $\downarrow$ \\
\hline & Attention & - & 0 & - & 0 & - & $\downarrow$ & 0 & 0 & $\downarrow$ \\
\hline & Verbal fluency & - & 0 & 0 & 0 & 0 & 0 & 0 & 0 & 0 \\
\hline & Processing speed & - & 0 & 0 & - & 0 & - & 0 & $\downarrow$ & 0 \\
\hline & Executive function & - & - & - & - & - & $\downarrow$ & 0 & - & 0 \\
\hline & Fine motor skills & - & - & + & - & - & - & - & - & - \\
\hline & Working memory & - & 0 & 0 & - & 0 & - & - & - & $\downarrow$ \\
\hline & Abstract reasoning & - & 0 & - & - & - & - & - & - & - \\
\hline & Naming & - & 0 & - & - & - & - & - & - & - \\
\hline
\end{tabular}

Abbreviation: $\mathrm{ADT}$, androgen deprivation therapy; $\mathrm{RCl}$, reliable change index.

X: Category/heading applies to study.

+ : Within subject improvement.

O: No effect.

$\uparrow \downarrow$ : Trending effect of ADT, or effect seen in one, but not all assessments.

$\uparrow \downarrow$ : Significant effect of ADT on cognitive domain (group differences).

$-:$ Not measured.

${ }^{a}$ Initial enrollment for each group not reported separately. Number reflects initial enrollment for all prostate cancer patients.

${ }^{\mathrm{b}}$ ADT group $<65$ years showed greater improvement following washout than controls.

*Significant difference between groups: healthy controls $>$ patients.

LHRH analog (leuprolid 11, $25 \mathrm{mg}$ subcutaneously, four times a year for 3 months) and concurrent radiotherapy. Fifty-two controls were also tested at baseline only, so the effects of ADT on cognition could not be ascertained in relation to what would be expected in the absence of $\mathrm{ADT}$ and prostate cancer. Cognitive function was assessed on a computerized test battery and occurred at baseline (i.e., pre-treatment in the LHRH group), 6 and 12 months during treatment. Healthy controls outperformed patients at baseline on tasks of verbal fluency, visuomotor abilities and sustained attention. Patients improved their performance on tasks of episodic and semantic memory after treatment. Given the lack of repeated testing of controls, it is unclear whether the magnitude of improvement is as great as would be expected in the absence of $\mathrm{ADT}$, since typically performance on the second assessment improves as a result of previous exposure to tests. Moreover, use of a computerized test battery makes comparisons to other studies challenging.

Mohile and Lacy ${ }^{42}$ examined changes in cognitive performance in a group of men (median age: 71 years) with asymptomatic metastatic prostate cancer. Cognitive testing was completed before patients began $\operatorname{ADT}(n=32)$ and 6 months following ADT initiation $(n=21)$. Cognitive tests included measures of language, verbal and visual memory, fine motor skills and executive function. At baseline, before and within 2 weeks of starting ADT, $45 \%$ of patients showed cognitive impairment on at least two cognitive tasks, where cognitive impairment was defined as scoring more than 1.5 standard deviation below the age and education-adjusted population reference means for that test. Evidence of baseline impairment was most evident on the Grooved Pegboard tests of fine motor speed $(60 \%$ and $90 \%$, respectively on the dominant and nondominant hand) and on the test of verbal memory ( $42 \%$ on verbal learning and delayed recall). Cognitive performance, as assessed by mean scores, did not change following ADT, except for fine motor skills which improved on the non-dominant hand. When assessed using RCI approaches, cognitive function changed following ADT, but with most showing mixed evidence of both improvement and decline across cognitive tests. Specifically, none of the subjects significantly declined, $14 \%$ significantly improved and 57\% showed a mix of both decline and improvement. This study suggests that men with asymptomatic metastatic prostate cancer may show cognitive declines before ADT treatment, though that conclusion is limited by the lack of an assessment of cognitive impairment in a control group.

Section summary. Overall, the three studies that followed patient groups only over time suggested that patients show improved cognitive performance over time and that their performance generally compares favorably with established norms. Two studies found evidence of 
cognitive impairment in patients before treatment with ADT, underscoring the importance of comparing patients to controls before treatment. ${ }^{40,42}$

\section{Observational studies comparing patients versus healthy controls over time}

Cherrier et al. ${ }^{47}$ conducted a prospective observational study involving a group of 19 men with non-metastatic prostate cancer in biochemical relapse treated with intermittent ADT (I-ADT) and a group of 15 healthy men (mean age of groups: 65 years). Patients were treated for 2 weeks with flutamide ( $250 \mathrm{mg}$, three times daily) or bicalutamide ( $50 \mathrm{mg}$ daily) if intolerant of flutamide followed by the addition of leuprolide (7.5 mg intramuscular) for a total of nine monthly doses. Cognitive assessments occurred twice before treatment, during the ninth month of treatment, and again after a 3-month washout. The second (pre-treatment) cognitive assessment served as baseline to minimize the influence of practice effects thereafter (since such effects are greatest from the first to second assessment). Cognitive measures included tests of spatial and verbal memory, spatial abilities, verbal fluency and attention. Results were analyzed separately for younger (51-66 years) and older (66-81 years) participants because age was a significant covariate in initial analyses. Among younger participants, there was an interaction between group and time on a measure of verbal memory (i.e., proactive interference) such that those treated with I-ADT showed a greater improvement in verbal memory following washout compared to controls (both groups performed similarly at baseline and 9 months). On the Route Test measure of spatial memory, the control group improved compared to baseline but the intermittent androgen suppression group did not. Based on RCI analyses, after androgen suppression, 69\% of the I-ADT group declined on spatial ability, but after treatment washout, only $15 \%$ of men in the I-ADT group continued to show decline. This I-ADT group also showed a significant improvement in verbal memory after washout. The number of controls showing impaired performance was not reported. Overall, this study indicates a decline in cognitive ability in men undergoing ADT with evidence of a return to normal function after treatment washout. The study weaknesses include small sample size, a lack of a control group of patients not receiving I-ADT, and challenges in controlling for age effects.

Jenkins et al. ${ }^{46}$ conducted a prospective observational study of 32 patients (mean age: 68 years) who were administered an LHRH analog as part of their routine medical care compared to healthy controls (mean age: 65 years). Cognitive assessments were timed to examine the shortand long-term effects of treatment by having participants complete assessments at baseline (pre-treatment), during the third month of treatment and after 9 months of treatment discontinuation (i.e., 9 months). Cognitive measures included tests of auditory/verbal memory, visual memory, verbal fluency, working memory and attention, processing speed and vigilance. There were no group differences on any task, including the Rey Auditory Verbal Learning Test, across all time points. RCI analyses revealed that $47 \%$ of patients declined on at least one cognitive task after 3 months of treatment with ADT compared to $17 \%$ of controls. Among the patients showing significant cognitive decline, $60 \%$ declined on a task of visuospatial ability (i.e., mental rotations, spatial memory or spatial working memory). Overall, this study provides evidence that a subset of men undergoing ADT may be vulnerable to cognitive decline associated with $\mathrm{ADT}$, specifically spatial memory.

Cherrier et al. ${ }^{48}$ examined the effects of 9 months of intermittent GnRHa (leuprolide) treatment and a daily anti-androgen (flutamide) pill in 19 hormone-naive, eugonadal prostate cancer patients with a rising PSA but with no signs of metastases (mean age: 62 years). The control group was healthy men (mean age: 66 years). Cognitive testing occurred at pre-baseline (before initiation of any treatment), baseline (again before initiation of any treatment), 3 months, 9 months (last treatment visit) and 12 months. Cognitive measures assessed a variety of domains including spatial ability, spatial memory, verbal memory, verbal fluency and executive function. After the 9-month assessment, ADT was stopped for a variable period of time. Three months after treatment, ADT patients performed worse than healthy controls on spatial memory, mental rotations and executive function tasks. These effects were not evident at the 9-month visit. Together, these findings suggest a transient decrease in spatial memory, mental rotations and executive function with ADT.

Section summary. Overall, consistent with findings from a previous review, ${ }^{12}$ these three rather small studies indicate an appreciably reliable finding of impaired spatial memory and spatial abilities in men receiving ADT for prostate cancer compared to healthy controls. These deficits appeared during the third month of treatment with ADT but were not found again at 9 months regardless of whether the patient was still receiving ADT. That finding may reflect the difficulties of detecting cognitive deficits after repeated cognitive testing, particularly in small sample sizes, or may suggest that any cognitive deficits are transient. Notably, there is good evidence that testosterone supplementation improves spatial memory and spatial abilities in men. $^{32,36,37,51,52}$

\section{Studies comparing patients on ADT to patients not on ADT to healthy controls}

The first study that directly compared the effects of ADT treatment on cognition in men with prostate cancer compared to untreated prostate cancer patients was published by Green and colleagues in $2004^{49}$ and built on a study first reported in $2002 .^{50}$ The earlier study will first be reviewed. It is significant because it represents the only study to date to directly compare different ADT treatments on cognitive function in men with prostate cancer, and does so in a randomized design.

Green's 2002 report $^{50}$ included 77 men (of 82 who consented) randomized to receive either leuprorelin (LHRH analog), goserelin (LHRH analog), cyproterone acetate (steroidal antiandrogen) or close clinical monitoring for 6 months. Cognitive assessments were completed at baseline and at 6-month follow up. Sixty-five of 77 patients randomized completed both cognitive assessments. The examiner was blinded to condition; it is unclear if patients were similarly blind to assignment. Cognitive domains assessed included memory, attention, and executive function for a total of 12 outcome measures. One notable omission was a test of spatial memory. Results revealed a significant group difference in performance across the two assessments on only one outcome, a measure of verbal learning (i.e., trials $1-5$ of the Rey Auditory Verbal Learning Test). Specifically, at 6 months, the men assigned to goserelin showed worse verbal learning compared to baseline, whereas men assigned to cyproterone showed improved verbal learning (there was a trend for the leuprorelin group to show a decrease on Trails B, a measure of processing speed and mental flexibility, compared to other groups). The RCI analyses revealed that men on ADT showed a higher frequency of significant cognitive decline than men on close clinical monitoring. The results were impressive; 24 of the 50 men on active treatments showed a reliable decline on at least one cognitive task compared with none in the close clinical monitoring group. Among the weaknesses of the study were that more than $20 \%$ of participants did not complete all cognitive tasks, sample sizes 
were somewhat small, groups showed baseline differences in IQ and education, it is unclear whether participants were blinded to condition, and missing data were replaced using inconsistent methods.

Green et al. ${ }^{49}$ published a follow-up study to the 2002 study in 2004 after adding a group of healthy community dwelling controls (mean age: 69 years) and a 12-month cognitive assessment. ${ }^{49}$ Cognitive assessments were completed at baseline and at 6- and 12-month follow-ups. Of 20 community dwelling controls, 15 completed all three cognitive assessments. Of the 82 men with prostate cancer, 62 (mean age: 73.5 years) completed all three assessments. Consistent with the 2002 publication, results indicated significant group differences in performance across the three assessments on the Rey Auditory Verbal Learning Test measure of verbal learning. At 6 months, the men assigned to goserelin showed poorer verbal learning compared with men assigned to leuprorelin (note that in the 2002 report, men assigned to cyproterone showed improved performance at 6 months). At the 1-year visit, men on ADT showed decreased verbal learning compared to men receiving close clinical monitoring, but there was no difference between the active treatment groups. Group differences over time were also observed on a measure of processing speed (i.e., the Digit Symbol Substitution Test). Men with no prostate cancer showed greater improvement over the three visits compared to men assigned to ADT treatments and to close clinical monitoring. Processing speed in men assigned to cyproterone acetate decreased from baseline. Overall, these data indicate that ADT may have a negative impact on verbal learning and processing speed in men with prostate cancer. Although this study benefits from the inclusion of a healthy control group and a longer follow-up, the weaknesses in the original publication remain.

The largest and best controlled study of the effects of ADT on cognitive function was published in 2010 by Alibhai and colleagues. ${ }^{13}$ Patients with prostate cancer starting ADT $(n=77)$, patients with prostate cancer not receiving $\mathrm{ADT}(n=82)$ and healthy controls $(n=82)$ performed cognitive tests at baseline, 6 months and 12 months. The mean age of the patients was 69 years and the range was 50-87 years. Importantly, the three groups were matched at baseline on important factors including age, education, global cognitive function and reading achievement. One weakness of the study is that the particular types of ADT were not specified. The cognitive test battery was comprised of well-validated, neuropsychological tests, with appropriate parallel forms where available for repeated testing. The cognitive domains assessed included: attention; processing speed; verbal fluency; visuospatial ability; verbal learning and memory; visual learning and memory; executive function/working memory; and executive function/cognitive flexibility. Note, however, that the battery did not include a sensitive spatial memory measure, like the Route Task or similar tasks shown in smaller studies to be impaired with ADT $^{46-48}$ or shown in clinical studies and randomized trials to improve with testosterone therapy in older men. ${ }^{36,37,51,52}$

Three different analytic approaches were used to examine the effects of ADT on cognition and were appropriate to examine given their use in the literature. One approach examined group differences in average scores. The other approaches were similar to the RCI approach but differed slightly; one examined group differences in the proportion of ADT patients scoring $\geqslant 1$ standard deviation compared to controls, and the third examined group differences in the proportion of participants who declined by more than 1.5 standard deviation on two or more tests. Results comparing group differences in mean scores revealed that after adjusting for age and education, ADT users showed worse immediate memory on one of two measures, working memory on one of four measures, and worse visuospatial abilities on one of two measures. Those findings were not evident in the RCI-like analyses. The authors concluded that there was no consistent evidence of deleterious cognitive effects of ADT over 12 months.

Section summary. The Alibhai investigation ${ }^{13}$ adds critically important new data to the literature, and incorporated the larger sample size and follow-up needed to better assess the effects of ADT on many aspects of cognition, including verbal memory. There were some minor weaknesses, including allowing baseline testing up to 4 weeks after starting ADT and use of a range of ADT treatments that were not clearly specified. From a cognitive test perspective, however, there was a significant weakness in the omission of a measure of spatial memory. The inclusion of such a task is critical given that spatial memory shows one of the largest and most reliable effects of testosterone in the literature. $^{36,37,46-48,51,52}$ The inclusion of a visual memory task, which measures memory for geometric designs, is informative, but the visual memory task does not show the sex differences or testosterone effects that a spatial memory task does. Moreover, given the importance of spatial navigation and memory in driving and other functional abilities, assessment of ADT in relation to spatial memory has important clinical significance.

Overall, the Green studies ${ }^{49,50}$ show notable difference from the Alibhai study ${ }^{13}$ in that a negative impact of ADT was observed on a test of verbal memory in the Green study but not the Alibhai study. The verbal memory measures used in the two 12-month studies were similar, though the earlier and shorter (i.e., 6-month) Green study pointed out different directions of change across verbal memory measures. The limited sample size and experimental weaknesses in the Green study, including baseline group differences in age and IQ, may account for the discrepancy. The sample size in the Green study ( $n=48$ on ADT versus $n=15$ not receiving ADT) was smaller than that in the Alibhai study ( $n=77$ on ADT and $n=82$ not receiving $\mathrm{ADT}$ ). On the other hand, Green and colleagues randomized participants to either close clinical monitoring or one of three forms of ADT, so the overall experimental design is stronger. Another possibility is that some of the particular ADT treatments used in the Green study have a more negative impact on verbal memory than those used in the Alibhai study. Specifically, Green found that goserelin had a more negative impact on verbal memory at 6 months than did leuprolin. ${ }^{49}$ It is possible that a larger proportion of $\mathrm{ADT}$-treated patients in the Green study received goserelin (19 of 48 men; 40\%) than in the Alibhai study. Unfortunately, the specific ADT treatments were not specified in the Alibhai study. On the other hand, Matousek and Sherwin examined the effects of goserelin treatment on verbal memory in men assessed before and after treatment, but no negative effects were evident. ${ }^{53}$

\section{DOES ESTRADIOL IMPROVE COGNITIVE FUNCTION IN MEN TREATED WITH ADT}

Beer and colleagues ${ }^{44}$ investigated cognitive function in 18 men diagnosed with androgen-independent prostate cancer (metastatic or PSA only) who were enrolled in a clinical trial of transdermal estradiol $\left(0.6 \mathrm{mg} \mathrm{d}^{-1}\right)$ as a second line hormonal therapy. Their cognitive performance was compared to 18 age-matched patients receiving ADT and 17 community dwelling healthy controls. The particular form of ADT differed among men, and groups differed in terms of mean vocabulary and overall global cognitive function at baseline. All groups were tested at baseline and then 4 weeks later, after the first group had discontinued ADT and received 4 weeks of transdermal estradiol treatment. At baseline, the two prostate cancer groups, both on ADT, 
performed worse on a measure of verbal story recall (both immediate and delayed), and showed slower processing speed compared with healthy community dwelling men. Men on ADT also showed more fatigue and confusion and less vigor compared with healthy control men. There was a near significant $(P=0.08)$ interaction between group and time such that performance on the on immediate story recall improved with treatment in the estradiol group $(P=0.08)$, but did not change in the other two groups. Estradiol treatment did not affect mood, but ADT negatively impacted confusion and depression. This study lends new insights into estradiol effects on cognition in men with prostate cancer and helped to justify further investigation into the potential benefits of estradiol on cognition in men with androgenindependent prostate cancer. Conclusions about ADT are limited by a lack of a pre-ADT baseline, by heterogenous ADT treatments (e.g., orchiectomy, GnHRa with or without antiandrogen therapy), and group differences in vocabulary and overall cognitive function.

Matousek and Sherwin ${ }^{53}$ conducted a randomized controlled trial of add-back estrogen or placebo on cognition in 21 men with prostate cancer. All patients first received an anti-androgen (Bicalutamide $50 \mathrm{mg} \mathrm{d}^{-1}$ ) for 2 weeks and then GnRHa. The specific GNRHa was chosen at the discretion of the patient's physician and was either goserelin acetate (10.8 mg s.c. every 12 weeks), leuprolide acetate (30 mg i.m. every 16 weeks) or leuprolide acetate for injectable suspension ( $22.5 \mathrm{mg}$ s.c. every 12 weeks). After 12 weeks, patients were randomized to receive estradiol or placebo. Patients were assessed at baseline (following anti-androgen treatment but before GnRHa), 12 weeks (ADT) and 24 weeks (estradiol versus placebo) on measures of verbal memory, spatial ability, visuomotor abilities and working memory). Results showed no significant within-subject change in performance from the assessment during anti-androgen treatment to the assessment following ADT. These results need to be interpreted with caution since there was no control group to assess rate of expected change in performance in the absence of treatment. After 24 weeks, the placebo group significantly outperformed the estradiol group on the immediate and delay portions of a verbal memory task (logical memory). Overall, this study suggests that estradiol may have negative effects on verbal memory in men treated with ADT.

\section{SUMMARY}

To date there have been 11 studies that examined the impact of ADT on cognitive performance. The most recent of these studies, the prospective study by Alibhai and colleagues, ${ }^{13}$ overcame a number of notable limitations in previous studies including small sample sizes, group differences in age and education at baseline, and lack of an untreated patient group. Thus, to date there is one high quality prospective observational study of the effects of ADT on cognition. The conclusions from that study are that ADT has little if any negative impact on cognitive function. Even that study, however, has a notable limitation in the omission of a test of spatial memory, which has been shown in the earlier studies to be especially sensitive to the effects of $\mathrm{ADT}$ and testosterone supplementation. ${ }^{32,36,37,51,52}$ Additionally, the specific types of ADT were not specified, so possible differences between therapies could not be evaluated, despite some suggestions from earlier studies that certain therapies (i.e., goserelin) may have more deleterious cognitive effects. Based on the available evidence, it seems that the effects of ADT on cognition may indeed be minimal, but there is need for caution in drawing this conclusion about ADT and spatial memory, since that particular cognitive domain was not assessed in the Alibhai study, despite a sizeable scientific literature documenting its particular sensitivity to ADT. ${ }^{32,36,37,51,52,54}$ There is mixed evidence of estrogen effects on cognition in men receiving ADT, with one study suggesting possible benefits to verbal memory ${ }^{44}$ and another suggesting possible decrements. ${ }^{53}$

Earlier studies have been critiqued for design weaknesses, including lack of estimation of practice effects, inconsistency of results, lack of effects across a number of domains, recruitment of well-educated samples that might not generalize to other samples, and use of methods that do not enable detection of subtle cognitive changes over time. ${ }^{13}$ These criticisms are appropriate for many studies, but do not apply to all studies. For example, all but three of the 11 studies controlled for practice effects by including a control group tested at similar intervals over time. ${ }^{46-48}$ Similarly, it is true that some studies found deficits in verbal memory ${ }^{46-48}$ and visuospatial memory and abilities, ${ }^{46-48}$ while others found no such evidence. On the other hand, each of three studies that assessed spatial memory found a significant negative impact of ADT on spatial memory. ${ }^{46-48}$ Thus, while some effects are variable, others appear to be quite robust. Similarly, it is wrong to assume that if ADT negatively impacts cognition, such effects should be evident across the majority of cognitive domains. Only a severe cognitive impairment, such as advanced Alzheimer's disease or severe traumatic brain injury, affects cognition globally. Sex steroid hormones affect only select areas of cognition, and especially affect cognitive domains subserved by brain regions where sex steroid hormone receptors are prominent. Basic science studies show that the effects of testosterone in the brain are limited to specific areas, particularly the hippocampus, and deficits are related to spatial abilities and encoding new memories. ${ }^{15,16}$ For that reason, it is to be expected that cognitive domains such as verbal memory and spatial abilities would be most sensitive to the effects of ADT. Criticisms regarding volunteer selection biases, particularly the educational level of subjects in the present literature, should be also be interpreted with caution. In the nine studies assessing only the effects of ADT on cognitive performance, the weighted average was 12.9 years of education. Participants in the Alibhai study averaged 16 years of education (college level) on average, while Green had 10.6 years (less than high school). Generalizing findings to less educated individuals remains a concern. A final criticism applies to most studies examining hormone effects on cognition, that is, concerns about the ability to detect subtle but persistent cognitive declines over time, in this case in men undergoing ADT. Detecting subtle cognitive impairments over time is problematic even with a valid and reliable battery of cognitive tests, because practice effects amass over time and obscure subtle cognitive effects. Practice effects are evident even in healthy individuals aged 70 years and older who are tested on two occasions 2 years apart. ${ }^{55}$ For this reason, it can be helpful to incorporate multiple baseline assessments, as Cherrier et al. ${ }^{48}$ has done in her ADT studies, to maximize practice effects before the estimation of treatment effects. It is notable that when such a design is used, the spatial memory effects are reliable. Neuroimaging studies may be helpful in detecting ADT effects over time. $^{55}$

The remaining gap in our understanding of the effects of ADT on cognition is the lack of a large-scale, randomized study comparing men randomized to ADT versus watchful waiting. Green conducted a small-scale study $(n=77)$ of this kind and found deleterious effects of ADT on verbal memory. ${ }^{49,50}$ On the other hand, Alibhai conducted a larger scale observational study $(n=241)$ and found no effects of ADT. From the available evidence, it appears most important to include tests of spatial memory, verbal memory and visuospatial abilities, and to a lesser extent processing speed. It would be helpful to incorporate these cognitive measures into future studies comparing ADT and watchful 
waiting on clinical progression of prostate cancer. Depending on future developments in the clinical literature, such a study might be possible, for example, in older men diagnosed by PSA within the early stages of prostate cancer. Additionally, it is important to consider what factors might contribute to vulnerability to negative cognitive effects of ADT, including increased age, low educational attainment and other cancer treatments.

\section{COMPETING FINANCIAL INTERESTS}

All authors declare that there are no competing financial interests.

1 Ferlay J, Shin HR, Bray F, Forman D, Mathers C et al. GLOBOCAN 2008, Cancer Incidence and Mortality Worldwide. IARC CancerBase No. 10. Lyon: International Agency for Research on Cancer; 2010. http://globocan.iarc.fr

2 Barry MJ, Delorenzo MA, Walker-Corkery ES, Lucas FL, Wennberg DC. The rising prevalence of androgen deprivation among older American men since the advent of prostate-specific antigen testing: a population-based cohort study. BJU Int 2006; 98 : 973-8.

3 Saylor PJ, Smith MR. Adverse effects of androgen deprivation therapy: defining the problem and promoting health among men with prostate cancer. J Nat/ Compr Canc Netw 2010; 8: 211-23.

4 Tammela T. Endocrine treatment of prostate cancer. J Steroid Biochem Mol Biol 2004; 92: 287-95

5 Shahinian VB, Kuo YF, Freeman JL, Orihuela E, Goodwin JS. Increasing use of gonadotropin-releasing hormone agonists for the treatment of localized prostate carcinoma. Cancer 2005; 103: 1615-24.

6 Barrass BJ, Thurairaja R, Persad RA. More should be done to prevent the harmful effects of long-term androgen ablation therapy in prostate cancer. BJU Int 2004; 93 : 1175-6.

7 Wilson LS, Tesoro R, Elkin EP, Sadetsky N, Broering JM et al. Cumulative cost pattern comparison of prostate cancer treatments. Cancer 2007; 109: 518-27.

8 Grossmann M, Zajac JD. Androgen deprivation therapy in men with prostate cancer: how should the side effects be monitored and treated? Clin Endocrinol 2011; 74: 289-93.

9 Taylor LG, Canfield SE, Du XL. Review of major adverse effects of androgendeprivation therapy in men with prostate cancer. Cancer 2009; 115: 2388-99.

10 Cancer Facts \&Figures 2011. Atlanta, GA: American Cancer Society; 2011.

11 Mathias JL, Lucas LK. Cognitive predictors of unsafe driving in older drivers: a metaanalysis. Int Psychogeriatr 2009; 21: 637-53.

12 Nelson CJ, Lee JS, Gamboa MC, Roth AJ. Cognitive effects of hormone therapy in men with prostate cancer: a review. Cancer 2008; 113: 1097-106.

13 Alibhai SM, Breunis H, Timilshina N, Marzouk S, Stewart D et al. Impact of androgendeprivation therapy on cognitive function in men with nonmetastatic prostate cancer. J Clin Oncol 2010; 28: 5030-7.

14 Finley SK, Kritzer MF. Immunoreactivity for intracellular androgen receptors in identified subpopulations of neurons, astrocytes and oligodendrocytes in primate prefrontal cortex. J Neurobiol 1999; 40: 446-57.

15 Leranth C, Petnehazy O, MacLusky NJ. Gonadal hormones affect spine synaptic density in the CA1 hippocampal subfield of male rats. J Neurosci 2003; 23: 1588-92.

16 Leranth C, Prange-Kiel J, Frick KM, Horvath TL. Low CA1 spine synapse density is further reduced by castration in male non-human primates. Cereb Cortex 2004; 14: 503-10.

17 Ramsden M, Nyborg AC, Murphy MP, Chang L, Stanczyk FZ et al. Androgens modulate beta-amyloid levels in male rat brain. J Neurochem 2003; 87: 1052-5.

18 Farrer LA, Cupples LA, Haines JL, Hyman B, Kukull WA et al. Effects of age, sex, and ethnicity on the association between apolipoprotein $E$ genotype and Alzheimer disease. A meta-analysis. APOE and Alzheimer Disease Meta Analysis Consortium. JAMA 1997; 278: 1349-56.

19 Raber J, Bongers G, LeFevour A, Buttini M, Mucke L. Androgens protect against apolipoprotein E4-induced cognitive deficits. J Neurosci 2002; 22: 5204-9.

20 Michael RP, Rees HD, Bonsall RW. Sites in the male primate brain at which testosterone acts as an androgen. Brain Res 1989; 502: 11-20.

21 Maki PM. A systematic review of clinical trials of hormone therapy on cognitive function: effects of age at initiation and progestin use. Ann NY Acad Sci 2005; 1052: 182-97.

22 Maki PM, Sundermann E. Hormone therapy and cognitive function. Hum Reprod Update 2009; 15: 667-81.

23 Morley JE, Kaiser FE, Perry HM 3rd, Patrick P, Morley PM et al. Longitudinal changes in testosterone, luteinizing hormone, and follicle-stimulating hormone in healthy older men. Metabolism 1997; 46: 410-3.

24 Harman SM, Metter EJ, Tobin JD, Pearson J, Blackman MR et al. Longitudinal effects of aging on serum total and free testosterone levels in healthy men. Baltimore Longitudinal Study of Aging. J Clin Endocrinol Metab 2001; 86: 724-31.

25 Feldman HA, Longcope C, Derby CA, Johannes CB, Araujo AB et al. Age trends in the level of serum testosterone and other hormones in middle-aged men: longitudinal results from the Massachusetts male aging study. J Clin Endocrinol Metab 2002; 87: 589-98.
26 Rosario ER, Chang L, Stanczyk FZ, Pike CJ. Age-related testosterone depletion and the development of Alzheimer disease. JAMA 2004; 292: 1431-2.

27 Moffat SD, Resnick SM. Long-term measures of free testosterone predict regional cerebral blood flow patterns in elderly men. Neurobiol Aging 2007; 28: 914-20.

28 Moffat SD, Zonderman AB, Metter EJ, Blackman MR, Harman SM et al. Longitudinal assessment of serum free testosterone concentration predicts memory performance and cognitive status in elderly men. J Clin Endocrinol Metab 2002; 87: 5001-7.

29 Moffat SD, Zonderman AB, Metter EJ, Kawas C, Blackman MR et al. Free testosterone and risk for Alzheimer disease in older men. Neurology 2004; 62: 188-93.

30 Hogervorst E, Williams J, Budge M, Barnetson L, Combrinck M et al. Serum total testosterone is lower in men with Alzheimer's disease. Neuroendocrinol Lett 2001; 22: $163-8$.

31 Janowsky JS, Chavez B, Orwoll E. Sex steroids modify working memory. J Cogn Neurosci 2000; 12: 407-14.

32 Janowsky JS, Oviatt SK, Orwoll ES. Testosterone influences spatial cognition in older men. Behav Neurosci 1994; 108: 325-32.

33 Sih R, Morley JE, Kaiser FE, Perry HM 3rd, Patrick P et al. Testosterone replacement in older hypogonadal men: a 12-month randomized controlled trial. J Clin Endocrinol Metab 1997: 82: 1661-7.

34 Kenny AM, Bellantonio S, Gruman CA, Acosta RD, Prestwood KM. Effects of transdermal testosterone on cognitive function and health perception in older men with low bioavailable testosterone levels. J Gerontol A Biol Sci Med Sci 2002; 57: M321-5.

35 Kenny AM, Fabregas G, Song C, Biskup B, Bellantonio S. Effects of testosterone on behavior, depression, and cognitive function in older men with mild cognitive loss. J Gerontol A Biol Sci Med Sci 2004; 59: 75-8.

36 Cherrier MM, Asthana S, Plymate S, Baker L, Matsumoto AM et al. Testosterone supplementation improves spatial and verbal memory in healthy older men. Neurology 2001; 57: 80-8.

37 Cherrier MM, Matsumoto AM, Amory JK, Ahmed S, Bremner W et al. The role of aromatization in testosterone supplementation: effects on cognition in older men. Neurology 2005; 64: 290-6.

38 Cherrier MM, Matsumoto AM, Amory JK, Johnson M, Craft S et al. Characterization of verbal and spatial memory changes from moderate to supraphysiological increases in serum testosterone in healthy older men. Psychoneuroendocrinology2007; 32: 72-9.

39 Haren MT, Wittert GA, Chapman IM, Coates P, Morley JE. Effect of oral testosterone undecanoate on visuospatial cognition, mood and quality of life in elderly men with low-normal gonadal status. Maturitas 2005; 50: 124-33.

40 Salminen E, Portin R, Korpela J, Backman H, Parvinen LM et al. Androgen deprivation and cognition in prostate cancer. Br J Cancer 2003; 89: 971-6.

41 Almeida OP, Waterreus A, Spry N, Flicker L, Martins RN. One year follow-up study of the association between chemical castration, sex hormones, beta-amyloid, memory and depression in men. Psychoneuroendocrinology 2004; 29: 1071-81.

42 Mohile SG, Lacy M, Rodin M, Bylow K, Dale W et al. Cognitive effects of androgen deprivation therapy in an older cohort of men with prostate cancer. Crit Rev Oncol Hematol 2010; 75: 152-9.

43 Bussiere JR, Beer TM, Neiss MB, Janowsky JS. Androgen deprivation impairs memory in older men. Behav Neurosci 2005; 119: 1429-37.

44 Beer TM, Bland LB, Bussiere JR, Neiss MB, Wersinger EM et al. Testosterone loss and estradiol administration modify memory in men. J Urol 2006; 175: 130-5.

45 Joly F, Alibhai SM, Galica J, Park A, Yi QL et al. Impact of androgen deprivation therapy on physical and cognitive function, as well as quality of life of patients with nonmetastatic prostate cancer. J Urol 2006; 176: 2443-7.

46 Jenkins VA, Bloomfield DJ, Shilling VM, Edginton TL. Does neoadjuvant hormone therapy for early prostate cancer affect cognition? Results from a pilot study. BJU Int 2005; 96: 48-53.

47 Cherrier MM, Rose AL, Higano C. The effects of combined androgen blockade on cognitive function during the first cycle of intermittent androgen suppression in patients with prostate cancer. J Urol 2003; 170: 1808-11.

48 Cherrier MM, Aubin S, Higano CS. Cognitive and mood changes in men undergoing intermittent combined androgen blockade for non-metastatic prostate cancer. Psychooncology 2009; 18: 237-47.

49 Green HJ, Pakenham KI, Headley BC, Yaxley J, Nicol DL et al. Quality of life compared during pharmacological treatments and clinical monitoring for non-localized prostate cancer: a randomized controlled trial. BJU Int 2004; 93: 975-9.

50 Green HJ, Pakenham KI, Headley BC, Yaxley J, Nicol DL et al. Altered cognitive function in men treated for prostate cancer with luteinizing hormone-releasing hormone analogues and cyproterone acetate: a randomized controlled trial. BJU Int 2002; 90: 427-32.

51 Cherrier MM, Craft S, Matsumoto $\mathrm{AH}$. Cognitive changes associated with supplementation of testosterone or dihydrotestosterone in mildly hypogonadal men: a preliminary report. J Androl 2003; 24: 568-76.

52 Cherrier MM, Matsumoto AM, Amory JK, Asthana S, Bremner W et al. Testosterone improves spatial memory in men with Alzheimer disease and mild cognitive impairment. Neurology 2005; 64: 2063-8.

53 Matousek RH, Sherwin BB. A randomized controlled trial of add-back estrogen or placebo on cognition in men with prostate cancer receiving an antiandrogen and a gonadotropin-releasing hormone analog. Psychoneuroendocrinology 2010; 35: 215-25.

54 Cherrier MM, Borghesani PR, Shelton AL, Higano CS. Changes in neuronal activation patterns in response to androgen deprivation therapy: a pilot study. BMC Cancer 2010; 10:1

55 Lamar M, Resnick SM, Zonderman AB. Longitudinal changes in verbal memory in older adults: distinguishing the effects of age from repeat testing. Neurology 2003; 60: 82-6. 